KANSAS JOURNAL of MEDICINE

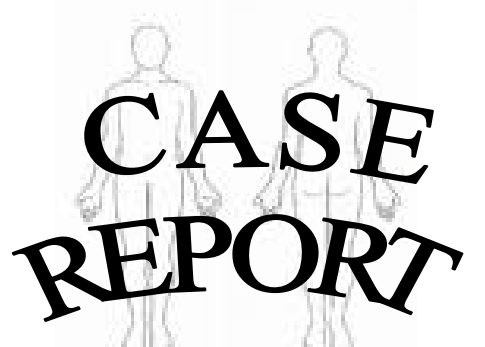

\section{Rhombencephalitis Presenting as a Manifestation of Neuropyschiatric Lupus}

Muhammad Imran, M.D. ${ }^{1}$, Ayesha Irum, M.S. ${ }^{2}$, Adam Parker, M.D. ${ }^{1}$, Leslie Kimpler, M.D. ${ }^{3}$, Phaedra Dowell, M.D. ${ }^{3}$, Anand Rajpara, M.D. ${ }^{4}$, Julian Magadan III, M.D. ${ }^{1}$

${ }^{1}$ University of Kansas Medical Center, Kansas City, KS Department of Internal Medicine, Division of Allergy, Clincial Immunology \& Rheumatology

${ }^{2}$ Army Medical College, Pawalpindi, Pakistan

University of Kansas Medicial Center, Kansas City, KS ${ }^{3}$ Department of Neurology

${ }^{4}$ Department of Internal Medicine, Division of Dermatology

\section{INTRODUCTION}

Systemic lupus erythematosus (SLE) is a chronic, relapsingremitting autoimmune disease which primarily affects the skin, joints, and kidneys but may involve any organ system, including peripheral, autonomic, or central nervous system (CNS). ${ }^{1}$ The CNS involvement may be considered primary if directly related to SLE activity or secondary when related to treatment complications, infections, or metabolic abnormalities such as uremia. ${ }^{2}$ The neuropsychiatric involvement in SLE (NPSLE), first mentioned by Kaposi more than 100 years ago, remains one of the main challenges facing the rheumatologist and other physicians. ${ }^{2}$

NPSLE can precede the onset of lupus or occur at any time during its course, most frequently within the first three years. ${ }^{3-6}$ The prevalence of CNS involvement in SLE ranges from $14 \%$ to $80 \%$, depending on the diagnostic criteria. ${ }^{7}$ SLE commonly involves the meninges, cranial nerves, cerebrum, spinal cord, and rarely involves the hindbrain, causing rhombencephalitis (RE), which is a syndrome of multiple causes and variable outcomes. The term "rhombencephalitis" refers to an inflammatory disease of the rhombencephalon or the hindbrain, which is composed of the pons, cerebellum, and the medulla oblongata. The term is derived from the Greek word, "rhombos" meaning a lozenge-shaped figure, plus "enkephalos", meaning the brain. ${ }^{8-10}$ No cases of SLE and rhombencephalitis were identified in a search of the medical literature.

\section{CASE REPORT}

The patient was a 43-year-old, right-handed, Caucasian female who presented to a local hospital several days after returning from a trip to Mexico. Her complaints included nausea, vomiting, urinary retention, headaches, and neck stiffness. The symptoms began suddenly while she was in Mexico, though she was not seen by a healthcare provider until she returned home. Her past medical history revealed she takes cetirizine as needed for seasonal allergies. She lived on a farm with her husband and two children. She denied any tobacco, alcohol, or illicit drug use. She had a family history of colon cancer; otherwise, family history was noncontributory.

She was admitted to the hospital at time of presentation and diagnosed with bacterial meningitis. She was treated empirically with vancomycin, ceftriaxone, and dexamethasone. She showed complete improvement clinically and was discharged home with a steroid taper and a total of 21 days of ceftriaxone.

The patient responded well until six months later when she again developed nausea, vomiting, diarrhea, and urinary retention. She was admitted to the same hospital for work-up. Magnetic resonance imaging (MRI) of the brain revealed a T2 hyperintensity at C2 - C3. A lumbar puncture revealed a red blood count of 2 UL, a white blood count of 63 UL with 31\% neutrophils and 30\% lymphocytes, glucose of $42 \mathrm{mg} / \mathrm{dl}$, and protein of $41 \mathrm{mg} / \mathrm{dl}$. Gram stain and culture on cerebrospinal fluid were negative. Other infectious studies also were negative, including West Nile, varicella zoster, and human immunodeficiency virus.

Rheumatologic serologic evaluation consisted of a positive antinuclear antibody test with titer 1:160 and positive anti-ds DNA. All other studies were negative, including rheumatoid factor, anti-Smith, anti-RNP, anti SSA/SSB, c-ANCA, and pANCA. The antiphospholipid antibodies were negative. Neuroinflammatory work-up revealed a negative neuromyelitis optica antibody, no oligoclonal bands on cerebrospinal fluid, and normal methylmalonic acid and serum protein electrophoresis.

The patient was diagnosed with transverse myelitis at the outside facility without having clear findings on MRI to support that diagnosis, which was based solely on symptoms of urinary retention. She was started on methylprednisolone, 1 gram for three days. Urinary retention resolved, but the patient remained fatigued. Steroids were discontinued and the patient was discharged home.

Noacuteeventsoccurred untileightweekslater when sheagain presented to her local hospital with nausea, vomiting, diarrhea, urinary retention, and fevers. Her temperature prior to presentation was $39.5^{\circ} \mathrm{C}$. Transverse myelitis was suspected again and the patient was transferred to our facility for further evaluation.

Upon presentation to our facility, the patient's exam was non-focal and unremarkable except erythematous rash on chest and back. She was alert and oriented to person, place, and time. Speech was intact for fluency, comprehension, articulation, repetition, and naming. Cranial nerves II-XII were intact. 


\section{KANSAS JOURNAL of MEDICINE}

\section{RHOMBENCEPHALITIS PRESENTING AS LUPUS}

continued.

Strength was 5/5 throughout; deep tendon reflexes were 2/4 throughout, and plantar response was down-going. Coordination was intact to finger-to-nose, heel-to-shin, and rapid alternating movements. Sensation was intact to light touch and pin prick; vibratory sensation was felt for more than 10 seconds at the bilateral great toes. Muscle tone and bulk were normal with no fasciculation, tremor, or pronator drift. Gait was normal and the patient was able to perform heel, toe, and tandem walk.

MRIs of the head and cervical spine revealed a fluid-attenuated inversion recovery (FLAIR) signal abnormality in the dorsal medulla and left lateral upper cervical spinal cord. This finding was not present on films performed at the outside facility. There was no MRI or clinical finding suggestive of transverse myelitis. Lumbar puncture revealed an opening pressure of $20 \mathrm{~cm} \mathrm{H}_{2} \mathrm{O}$, red blood cell count of 30/UL, white blood cell count of $80 / \mathrm{UL}$ with $6 \%$ neutrophils and $56 \%$ lymphocytes, glucose of $35 \mathrm{mg} / \mathrm{dl}$, and protein of $48 \mathrm{mg} / \mathrm{dl}$.

Clinically, the patient did not have signs of infection. Her fever had resolved. An infectious disease specialist was consulted and agreed with holding antibiotics at that time. Fever returned with nightly spikes to greater than $103^{\circ} \mathrm{F}$. She was treated with acetaminophen and fever resolved with one dose. The patient required frequent catheterization to empty her bladder. All other symptoms persisted.

Lumbar puncture was repeated two days after admission and revealed opening pressure of $8 \mathrm{~cm} \mathrm{H}_{2} \mathrm{O}$, red blood cell count of 0 , white blood cell count of 380 with $63 \%$ neutrophils and 20\% lymphocytes, glucose of $32 \mathrm{mg} / \mathrm{dl}$, and protein of $69 \mathrm{mg} / \mathrm{dl}$. Oligoclonal bands were negative. In light of this increase in white blood cell count, MRI findings in the brainstem, and return of fever, the patient was started empirically on ampicillin for Listeria coverage as it was suspected as the most likely organism contributing to her rhombencephalitis.

Her fevers continued cyclically with spikes of greater than $102^{\circ} \mathrm{F}$ overnight every night. Rheumatologic workup revealed antinuclear antibodies of 380 with nucleolar pattern, complement C3 of $19.6 \mathrm{mg} / \mathrm{dl}$, complement C4 of greater than $5.0 \mathrm{mg} / \mathrm{dl}$, complement $\mathrm{CH} 50$ of $3 \mathrm{AU}$, antiSmith positive, anti-RNP positive, anti-SSA and SSB negative, and ds-DNA positive. $\operatorname{IgM}$, IgA, and $\operatorname{IgG}$ were within normal limits. Histoplasma antigen also was negative.

The patient had an erythematous rash on her back and chest throughout admission. The patient reported a similar rash had accompanied her two prior episodes as well, which would improve once steroids were initiated. The rash was described as a v-shaped, photo-distributed erythematous area with scaly papules on the anterior chest (Figure 1). A punch biopsy was consistent with cutaneous lupus erythematosus (Figure 2). The v-shape rash and interface dermatitis can be seen in dermatomyositis, but the patient had no muscle weakness, Gottron's papules, and heliotrope rash on physical examination and her CPK, aldolase and anti Jo were negative.

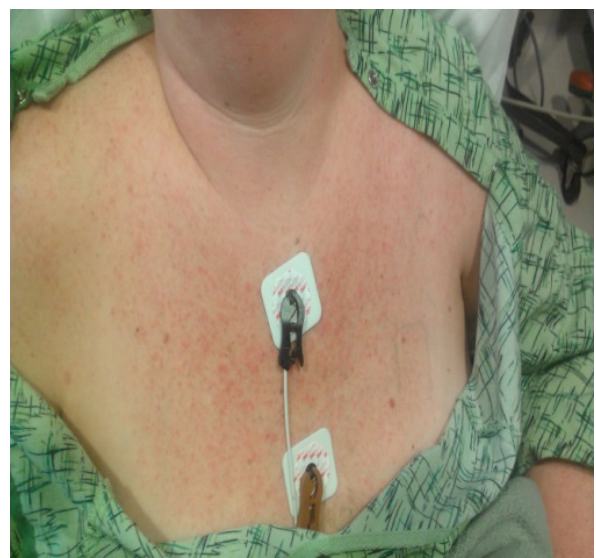

Figure 1. A v-shaped, photo-distributed erythematous area with scaly papules on the anterior chest.

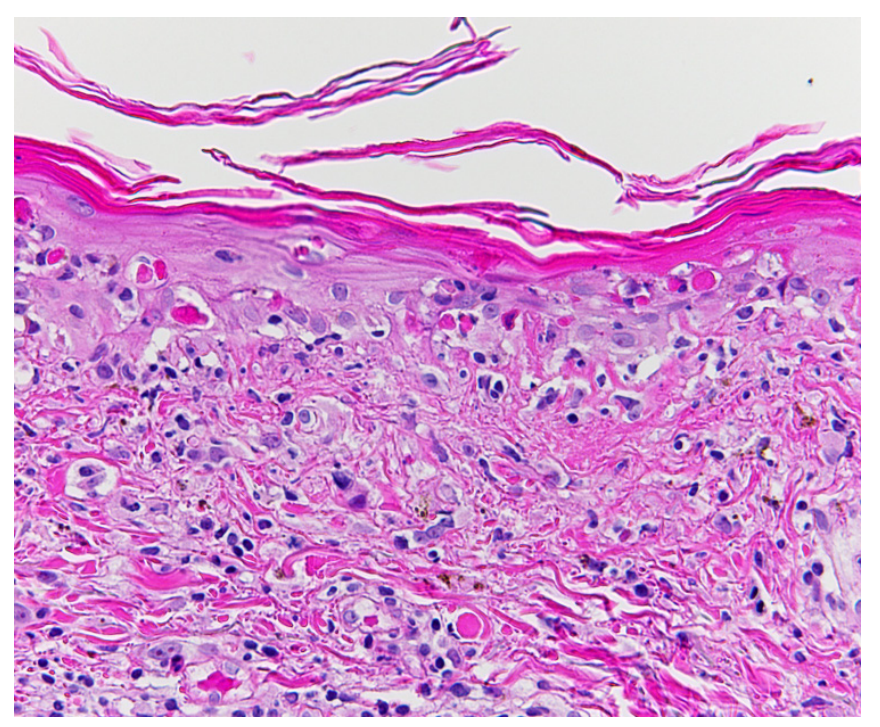

Figure 2. Vacuolar interface dermatitis with scale, epidermal thinning and scattered necrotic keratinocytes in the epidermal spinous layer; basal layer vacuolization and a dermal infiltrate of lymphocytes, histiocytes and melanophages (hematoxylin-eosin, X200).

Lumbar puncture was repeated and revealed an opening pressure of $12.5 \mathrm{~cm} \mathrm{H}_{2} \mathrm{O}$, red blood cell count of 53/UL, white blood cell count of 100/UL with 19\% neutrophils and 57\% lymphocytes, glucose of $55 \mathrm{mg} / \mathrm{dl}$, and protein of $176 \mathrm{mg} / \mathrm{dl}$. Ampicillin was discontinued in light of these results as well as no appreciable improvement in cerebrospinal fluid white blood cell count after one week of treatment. The patient was started on prednisone for systemic lupus erythematosus and discharged home.

Since discharge, the patient has been followed closely as an outpatient. Disease modifying anti-rheumatic drug therapy (DMARD) was initiated with mycophenolate mofetil 1,000 $\mathrm{mg}$ twice a day and hydroxychloroquine $200 \mathrm{mg}$ twice a day. She improved clinically and has been tapered off prednisone.

\section{DISCUSSION}

Rhombencephalitis (RE) has a wide variety of etiologies, some potentially severe and life threatening without proper early diagnosis and treatment. The etiologies 
KANSAS JOURNAL of MEDICINE

RHOMBENCEPHALITIS PRESENTING AS LUPUS

continued.

include infections, autoimmune diseases, and paraneoplastic syndromes. ${ }^{2}$ The most common infectious etiologies include Listeria, enterovirus 71, and herpes viruses, while the most common autoimmune etiology is Behçet's disease. RE is seen in SLE and relapsing polychondritis. ${ }^{11-13}$ The exact pathogenesis is not known, but large numbers of pathophysiologic processes are hypothesized to be involved, including anti-neuronal antibodies, antibodies against ribosomal P-protein, cytokines, vascular injury induced by circulating immune complex, occlusive vasculopathy as a result of endothelial cell activation induced by cytokines and complement activation, or macroand microvascular thrombosis induced by antiphospholipid antibodies. In the later stages of disease, cerebrovascular manifestations often are related to accelerated atherosclerosis., ${ }^{1,14}$ However, direct and unequivocal evidence for the implication of any of the above-mentioned mechanisms is lacking.

The clinical features of RE include altered mental status, hallucination, headache, unilateral cranial nerve paresis (mainly V, VII, VI, IX, and X), cerebellar deficits (hemiataxia, vertigo, or dysarthria), respiratory failure, dysphagia, quadriplegia, ocular movement dysfunction, meningitis, and encephalopathy. ${ }^{16-18}$ Our patient had headache followed by the development of encephalopathy. There is no single test laboratory or imaging finding which is diagnostic for NPSLE. The diagnosis is established based on the constellation of clinical presentation, serologic tests, and neuroimaging techniques which are used to exclude other potential etiologies prior to the diagnosis of SLE related RE.

Our patient did not have another possible explanation for $\mathrm{RE}$ and her serologic testing was supportive of a new diagnosis of SLE (SLICC classification criteria: cutaneous lupus, positive ANA, positive anti-ds DNA, and hypocomplementemia). The serologic studies revealed elevated anti-double stranded DNA and hypocomplementemia, suggestive of active disease. The cerebrospinal fluid analysis in CNS lupus usually presents with lymphocytic pleocytosis and elevated protein. ${ }^{19}$ MRI of the brain is the imaging modality of choice in case of CNS lupus, especially rhombencephalitis. The usual MRI findings in rhombencephalitis are increased signal intensity in the pons, medulla, upper cervical cord, and cerebellum more frequently than the midbrain on T2-weighted images. ${ }^{20}$

Therapy usually consists of high-dose intravenous corticosteroids followed by slow oral tapering doses. ${ }^{21,22}$ The most commonly used steroid sparing agents in these cases are azathioprine or cyclophosphamide. Less frequently used immunosuppressive agents include cyclosporine, methotrexate, and anti-tumor necrosis factor alpha (TNF- $\alpha$ ) agents. ${ }^{20-23}$ The most commonly used steroid sparing agents are azathioprine, mycophenolate mofetil, or cyclophosphamide. Mycophenolate mofetil was chosen given its more favorable side ef- fect profile compared to cyclophosphamide and case reports of aseptic meningitis in association with azathioprine..$^{23-26}$

Some poor prognostic factors include repeated attacks, incomplete recovery, progression of disease, and a high level of CSF pleocytosis during the acute attack. ${ }^{24,26}$ About $25 \%$ of patients have complete recovery, while $75 \%$ have residual motor, sensory, visual, and cognitive impairments.

\section{CONCLUSION}

SLE may have variable CNS manifestations. SLE should be considered as a potential cause of rhombencephalitis. MRI is useful in demonstrating brain lesions and in evaluating treatment efficacy.

\section{REFERENCES}

${ }^{1}$ Scolding NJ, Joseph FG. The neuropathology and pathogenesis of systemic lupus erythematosus. Neuropathol Appl Neurobiol 2002; 28(3):173-189. PMID: 12060342. 2 Johnson RT, Richardson EP. The neurological manifestations of systemic lupus erythematosus. Medicine (Baltimore)1968; 47(4):337-369. PMID: 5212395. ${ }^{3}$ Bluestein HG. Neuropsychiatric disorders in systemic lupus erythematosus. In: RG Lahita (Ed). Systemic Lupus Erythematosus. New York: John Wiley and Sons, 1986. ${ }^{4}$ Borchers AT, Aoki CA, Naguwa SM, Keen CL, Shoenfeld Y, Gershwin ME. Neuropsychiatric features of systemic lupus erythematosus. Autoimmun Rev 2005; 4(6):329-344. PMID: 16081024. ${ }^{5}$ Unterman A, Nolte JE, Boaz M, Abady M, Shoenfeld Y, Zandman-Goddard G. Neuropsychiatric syndromes in systemiclupus erythematosus: A meta-analysis. Semin Arthritis Rheum 2011; 41(1):1-11. PMID: 20965549. ${ }^{6}$ Hanly JG, McCurdy G, Fougere L, Douglas JA, Thompson K. Neuropsychiatric events in systemic lupus erythematosus: Attribution and clinical significance. J Rheumatol 2004; 31(11):2156-2162. PMID: 15517627.

${ }^{7}$ Sanna G, Bertolaccini ML, Khamashta MA. Neuropsychiatric involvement in systemic lupus erythematosus: Current therapeutic approach. Curr Pharm Des 2008; 14(13):1261-1269. PMID: 18537650.

${ }^{8}$ Armstrong RW, Fung PC. Brainstem encephalitis (rhombenecephalitis) due to Listeria monocytogenes: Case report and literature review. Clin Infect Dis 1993; 16(5):689-702. PMID: 8507761. 9 Smiatacz T, Kowalik MM, Hlebowicz M. Prolonged dysphagia due to Listeria-rhombencephalitis with brainstem abscess and, acute polyradiculoneuritis. J Infect 2006; 52(6):e165-e167. PMID: 16260041. ${ }_{10}$ Crawford JR, Kadom N, Santi MR, Mariani B, Lavenstein BL. Human herpesvirus 6 rhombencephalitis in immunocompetent children. J Child Neurol 2007; 22(11):1260-1268. PMID: 18006954. ${ }^{11}$ Antal EA, Dietrichs E, Løberg EM, Melby KK, Maehlen J. Brain stem encephalitis in listeriosis. Scand J Infect Dis 2005; 37(3):190-194. PMID: 15849051. ${ }_{12}$ Wadia N, Williams E. Behcet's syndrome with neurological complications. Brain 1957; 80(1):59-71. PMID: 13412995. ${ }_{13}$ Wasserfallen JB, Schaller MD. Unusual rhombencephalitis in relapsing polychondritis. Ann Rheum Dis 1992; 51(10):1184. PMID: 1444642. ${ }^{14}$ Hanly JG, Walsh NM, Sangalang V. Brain pathology in systemic lupus erythematosus. J Rheumatol 1992; 19(5):732-741. PMID: 1613703. ${ }^{15}$ Hahn BH, Grossman J, Chen W, McMahon M. The pathogenesis of atherosclerosis in autoimmune rheumatic diseases: Roles of inflammation and dyslipidemia. J Autoimmun 2007; 28(2-3):69-75. PMID: 17433865. ${ }_{16}$ Moragas M, Martínez-Yélamos S, Majós C, Fernández-Viladrich P, Rubio F, Arbizu T. Rhombencephalitis: A series of 97 patients. Medicine (Baltimore) 2011; 90(4):256-261. PMID: 21694648. ${ }^{17}$ Jubelt B, Mihai C, Li TM, Veerapaneni P. Rhombencephalitis/brainstem encephalitis. Curr Neurol Neurosci Rep 2011; 11(6):543-552. PMID: 21956758. ${ }^{18} \mathrm{Kamm}$ C, Zettl UK. Autoimmune disorders affecting both the central and peripheral nervous system. Autoimmun Rev 2012; 11(3):196-202. PMID: 21619947. ${ }^{19}$ Graham JW, Jan W. MRI and the brain in systemic lupus erythematosus. Lupus 2003; 12(12):891-896. PMID: 14714907. ${ }_{20}^{20}$ Trevisani VF, Castro AA, Neves Neto JF, Atallah AN. Cyclophosphamide versus methylprednisolone for treating neuropsychiatric involvement in systemic lupus erythematosus. Cochrane Database Syst Rev 2006; (2):CD002265. PMID: 16625558. 


\section{KANSAS JOURNAL of MEDICINE}

\section{RHOMBENCEPHALITIS PRESENTING AS LUPUS}

continued.

${ }^{21}$ Barile-Fabris L, Ariza-Andraca R, Olguín-Ortega L, et al. Controlled clinical trial of IV cyclophosphamide versus IV methylprednisolone in severe neurological manifestations in systemic lupus erythematosus. Ann Rheum Dis 2005; 64(4):620-625. PMID: 15769918. ${ }_{22}^{2}$ Fortin PR, Abrahamowicz M, Ferland D, et al. Steroidsparing effects of methotrexate in systemic lupus erythematosus: A double-blind, randomized, placebo-controlled trial. Arthritis Rheum 2008; 59(12):1796-1804. PMID: 19035431. ${ }^{23}$ Blank M, Shoenfeld Y. B cell targeted therapy in autoimmunity. J Autoimmun 2007; 28(2-3):62-68. PMID: 17391915.

${ }^{24}$ Castellino G, Govoni M, Giacuzzo S, Trotta F. Optimizing clinical monitoring of central nervous system involvement in SLE. Autoimmun Rev 2008; 7(4):297-304. PMID: 18295733.

${ }^{25}$ Gonzales-Crespo MR, Blanco FJ, Ramos A, et al. Magnetic resonance imaging of the brain in systemic lupus erythematosus. Br J Rheumatology 1995; 34(11):1055-1060. PMID: 8542207. ${ }^{26}$ Metreau-Vastel J, Mikaeloff Y, Tardieu M, Koné-Paut I, Tran TA. Neurological involvement in paediatric Behçet's disease. Neuropediatrics 2010; 41(5):228-234. PMID: 21210339.

Keywords:

neuropsychiatric

system-

ic lupus

erythematosus,

rhombencephalon,

inflammation 